\title{
Comparison of Voltage Map Calculation Methods Using Non-Fractionated EGM Signals in a Persistent AF Patient
}

\author{
Deborah Nairn $^{1}$, Heiko Lehrmann ${ }^{2}$, Amir Jadidi $^{2}$, Olaf Dössel $^{1}$, Axel Loewe $^{1}$ \\ ${ }^{1}$ Institute of Biomedical Engineering, Karlsruhe Institute of Technology (KIT), Karlsruhe, Germany \\ ${ }^{2}$ Universitäts-Herzzentrum Bad Krozingen, Bad Krozingen, Germany
}

\begin{abstract}
Catheter ablation targeting low voltage areas (LVA) is commonly being used to treat atrial fibrillation $(A F)$ in patients with persistent AF. However, it is not always certain that the areas marked as low voltage (LV) are correct. This can be related to how the voltage is calculated. Therefore, this paper focuses on comparing different calculation methods, specifically, with regards to spatial distribution.

Two voltage maps obtained in AF were used, removing points which did not meet the required specifications. The peaks for the remaining points, in regions of the left atrium, were then found and the voltage was calculated based on taking the peak to peak ( $p 2 p)$ for different beats.

For around $30 \%$ of the points on the map, the voltage only changed by $0.1 \mathrm{mV}$ when taking one beat versus all beats. However, for some individual points, the difference was substantial, around $0.8 \mathrm{mV}$, depending on the beat chosen. Additionally, the inter-method variability increased by around $0.1 \mathrm{mV}$ when considering all methods compared to only methods calculated using more than one point.

It was found that taking the median over all $p 2 p$ values in each point would be a more appropriate method for calculating the voltage. Thus, providing a technique, which could improve the accuracy of identifying LVA in an AF map.
\end{abstract}

\section{Introduction}

Atrial fibrillation (AF) is a common cardiac arrhythmia characterised by an irregular heart rhythm, which can cause various complications such as heart failure and stroke [1]. It is therefore very important to treat this arrhythmia. One technique is pulmonary vein isolation, which has become widely used to provide freedom from AF, especially in paroxysmal AF $[2,3]$. However, in persistent $\mathrm{AF}$ this technique alone is not always successful, with variation occurring between patient success rates [4]. It has been seen in many studies, that additionally targeting low voltage areas (LVA) can significantly improve the out- come of ablation [5-7]. Currently, work is being done to correlate the voltage maps between sinus rhythm (SR) and AF [8]. However, with the noise and irregular rhythm that is associated with $\mathrm{AF}$, it remains unclear if the voltage provided in the map has been calculated robustly. Therefore, it must be determined whether there exists an appropriate method for voltage calculation to accurately locate the areas of LV.

In this paper, it will be investigated how different calculations methods relate to one another. Specifically assessing the difference in voltage when only one beat in the bipolar signal is used in comparison to the whole signal. Additionally, ten regions of the left atrium will be analysed to understand whether inter-method variability may be region-dependent.

\section{Methods}

For this study, $2.5 \mathrm{sec}$ electrocardiogram (ECG) and bipolar electrogram (EGM) signals were provided by the CARTO3 system, allowing the voltage to be calculated in different ways on each point of the induced AF map. To ensure the accuracy of the data being used, certain considerations were made to determine which points would be analysed. This consisted of using the tissue proximity indicator on CARTO to ensure that all points included in the analysis were obtained with sufficient contact to the tissue.

Prior to calculating the voltage for each point, the QRS area in the corresponding ECG was identified. Therefore, the voltage is only calculated on beats in a window before the QRS complex to ensure information from the ventricles was not included. Using a similar approach to the PanTompkin's QRS detection algorithm, zero crossings of the derived ECG and a template matching algorithm, the QRS area was identified [9].

The peaks used for the voltage were then found in these windows, based again on the Pan Tompkin's algorithm which instead of the differentiation, squaring and movingwindow integration used a Non-Linear Energy Operator (NLEO) and a Gaussian Lowpass filter [10]. Additionally, points on the map in which no peak was found with an 
amplitude over $0.05 \mathrm{mV}$ were removed from the analysis as the signals appeared to be very noisy. Moreover, if the time stamp indicated that the catheter was moved during the $2.5 \mathrm{sec}$ signal, a part of the signal was removed to avoid problems due to motion artefact.

Furthermore, as it is not easy to determine which peaks should be used for evaluation in fractionated signals, the complex fractionated atrial electrograms (CFE) mean was calculated for each point, using a similar method to that of NavX. All signals with a CFE mean $<80 \mathrm{~ms}$ were then considered as continuously fractionated and removed from the analysis [11].

The voltage provided by CARTO was obtained by taking the peak to peak ( $\mathrm{p} 2 \mathrm{p}$ ) value (local maximum - local minimum) of one beat. Although this seems adequate for SR since there is not a lot of variation in the signal, it is important to identify if this is also the case in AF. Therefore, for each point, the mean and median over all the $\mathrm{p} 2 \mathrm{p}$ values of the signal were calculated, to allow a direct comparison between taking the whole signal and taking only one beat. Moreover, to further examine how the voltage values change with different methods, the maximum and minimum of the $p 2 p$ values were also calculated. The 75 th percentile of the $\mathrm{p} 2 \mathrm{p}$ beats was also taken to identify a method which would give the voltage calculated on beats with higher amplitudes but without including the outliers, like the max and min. Finally, the points of the left atrium were separated into ten regions by k-means clustering to correlate the voltage with its spatial distribution.

\section{Results}

Two patients in persistent AF undergoing their first ablation were used for this study, with a total of 4241 and 2436 mapping points, respectively. After the removal of the points which did not meet the criteria, as mentioned above, 3076 and 1223 were left for analysis.

When assessing the voltage values at different points, it was seen that calculating the voltage on one beat can give very different values to using the same way but over all beats. In Figure 1, this can be seen in the form of a bar chart, where the voltage is calculated using the different p2p methods described above. Each of the first four bars are calculated using only one beat, the following two bars are then calculated by taking the mean and median over the four $\mathrm{p} 2 \mathrm{p}$ values. Finally, the last bar is the value provided by the CARTO system which was also calculated from $\mathrm{p} 2 \mathrm{p}$ over one beat.

To further understand the values being provided in the bar chart, the $2.5 \mathrm{sec}$ signal of that point can be seen in Figure 2, with the QRS regions set to zero, so that the areas which were used for analysis are more defined.

It can be seen by the first figure that taking only one beat to calculate the voltage can give a significantly different

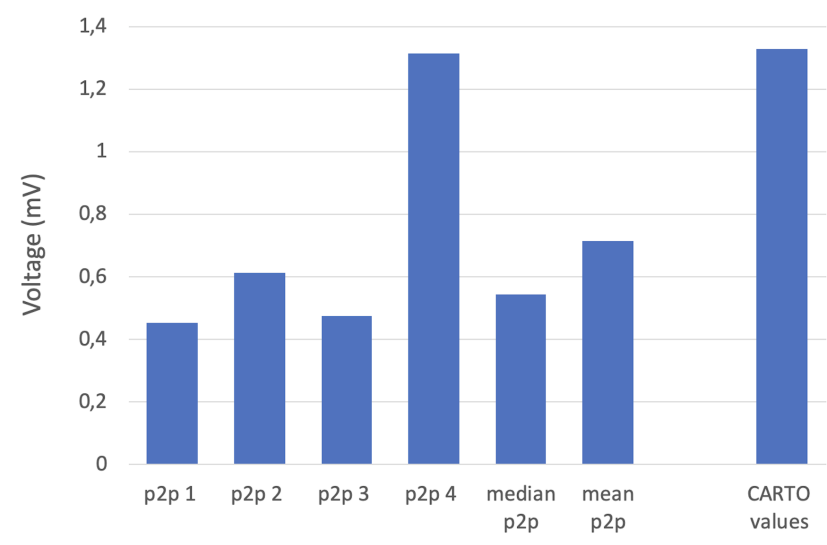

Figure 1. Bar chart showing the voltage calculated for one point in one of the maps with different beats of the signal

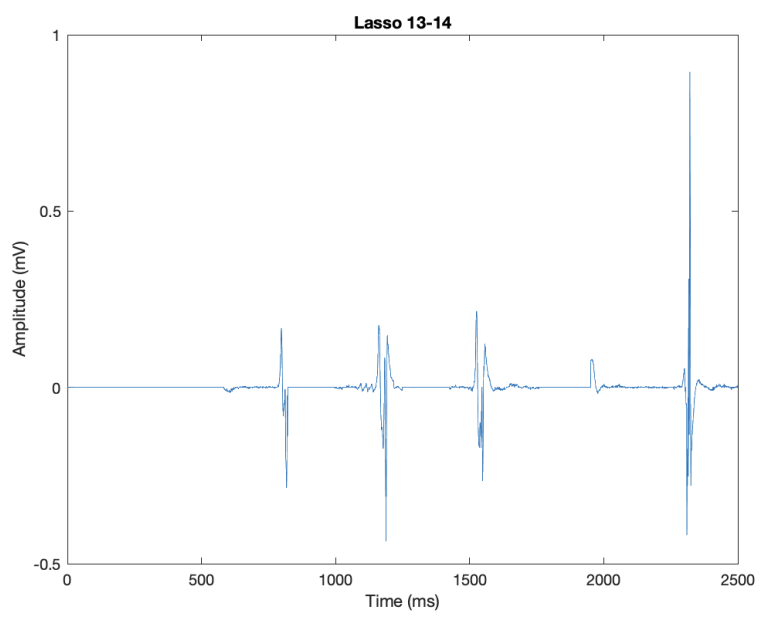

Figure 2. (2.5 sec signal of the same point on the map, as in Figure 1, with the information in the QRS region set to 0

value than if the voltage was calculated over all the beats. However, it must be assessed if this is true for all points on both maps. Therefore, for each method mentioned above, the mean and standard deviation of the voltage values were calculated over all points. Additionally, the absolute difference between each method and the median $\mathrm{p} 2 \mathrm{p}$ method for each point was calculated and the mean was taken over all points. Figure 3 shows a bar chart where the bars represent the mean value of the voltage and absolute difference for each method and the error bars represent the standard deviation.

When comparing the median and CARTO value in Figure 2 a difference of $0.8 \mathrm{mV}$ is seen. However, in Figure 3 there is an average difference of $0.2 / 0.3 \mathrm{mV}$. Therefore, it can be seen that the situation for that one point is not the situation for all points but in general a change is occurring when calculating the voltage using only one beat. 


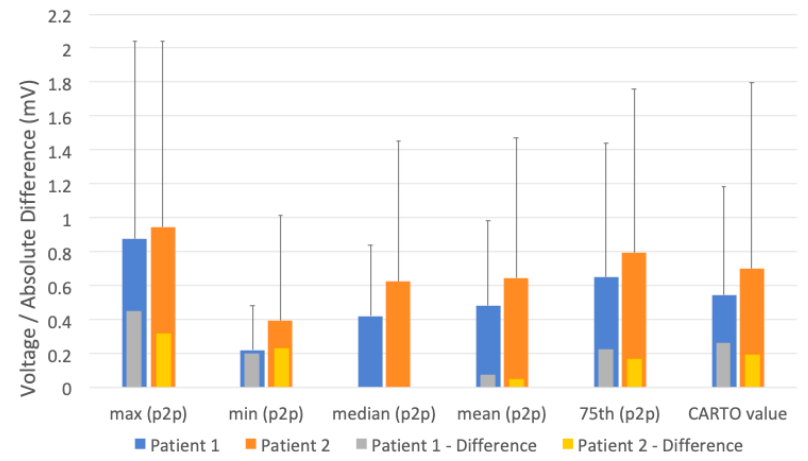

Figure 3. Bar chart with error bars showing the mean and standard deviation of the voltage value and the absolute difference between each method and the median $p 2 p$ value for both patients

This is further verified by all the methods having an average difference of around $0.2 \mathrm{mV}$ except the mean $\mathrm{p} 2 \mathrm{p}$ when compared to the median.

To assess more clearly how many points have a similar voltage when calculated with median $\mathrm{p} 2 \mathrm{p}$ to that of the CARTO value, Table 1 was created. This shows that when comparing the voltage calculated by the median $\mathrm{p} 2 \mathrm{p}$, around $30 \%$ of points were in a $0.1 \mathrm{mV}$ range from the CARTO value. Although $0.1 \mathrm{mV}$ does not seem a substantial difference, areas which were classed as LVA may no longer be, or vice versa. The columns Max and Min present the same results but instead of comparing the median with the CARTO value, they are compared with the $\max p 2 p$ and min $p 2 p$ values respectively. The percentage of points with similar values to the median $\mathrm{p} 2 \mathrm{p}$ appears lower than with the CARTO value, with around $21.5 \%$ of points having a $0.1 \mathrm{mV}$ difference between the median $\mathrm{p} 2 \mathrm{p}$ and the max $\mathrm{p} 2 \mathrm{p}$.

Table 1. Percentage of points for which the absolute difference in voltage values between using one beat and the whole signal is less than the difference given

\begin{tabular}{|c|ccc|ccc|}
\hline Comparison & \multicolumn{3}{|c|}{ Patient 1 } & \multicolumn{3}{c|}{ Patient 2 } \\
\hline Difference (mV) & CARTO & Max & Min & CARTO & Max & Min \\
0 & $23 \%$ & $22 \%$ & $22 \%$ & $31 \%$ & $25 \%$ & $26 \%$ \\
0.1 & $31 \%$ & $21 \%$ & $25 \%$ & $29 \%$ & $22 \%$ & $26 \%$ \\
0.2 & $17 \%$ & $13 \%$ & $17 \%$ & $15 \%$ & $13 \%$ & $13 \%$ \\
0.3 & $8 \%$ & $10 \%$ & $12 \%$ & $9 \%$ & $10 \%$ & $10 \%$ \\
\hline
\end{tabular}

In Figure 4 the same methods for calculating the voltage are analysed, with the added aspect of looking at the spatial distribution. The left atrium was split into ten sections for both patients and the average median voltage for each section of patient 2 are shown. The line graph in Figure 5 then shows the average voltage in each section for the different calculation methods for the same patient. Addition-
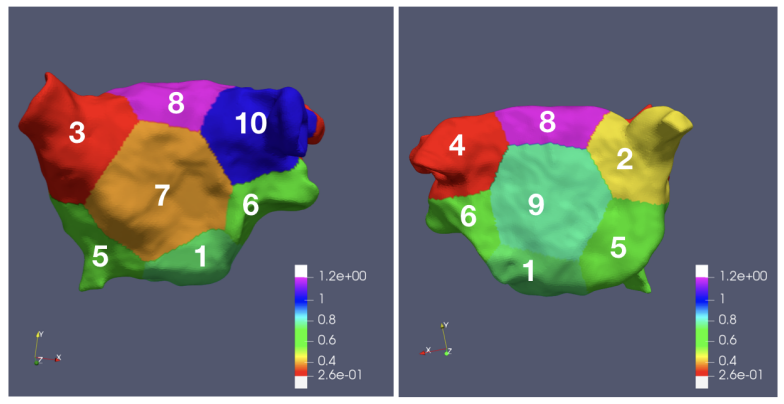

Figure 4. Image showing the left atrium split into ten sections with the average median voltage given for each section

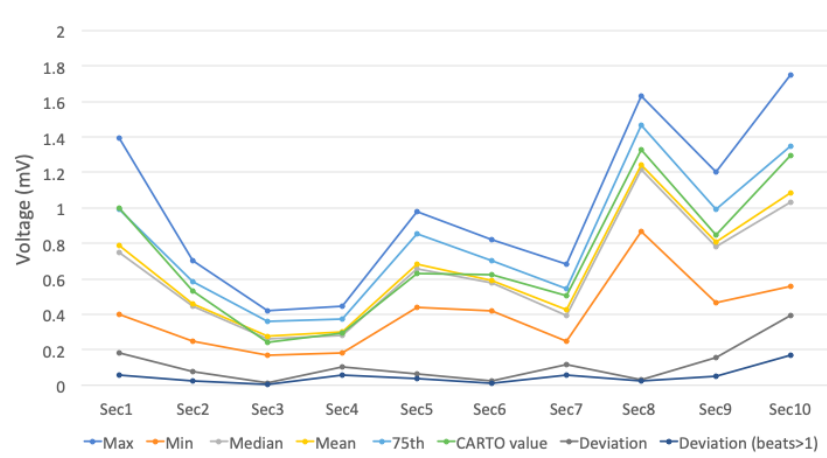

Figure 5. Line graph showing the average voltage for each section and method and the standard deviation between methods

ally, the standard deviation between the methods for each section is given, along with the standard deviation between only methods where more than one beat was used for the calculation.

From Figure 5 it can be seen that the voltage is changing depending on which area of the atrium is being looked at, which can be due to the thickness of the tissue or areas of diseased tissue. However, the variation between methods remains relatively constant between sections, except for the CARTO method which causes most of the variation in the deviation line. When considering the variation between only methods calculated using more than one beat, the average standard deviation is $0.05 \mathrm{mV}$, which raises to $0.13 \mathrm{mV}$ when all methods are included. Patient 1 also shows similar results with an increase of $0.1 \mathrm{mV}$ between calculating the variation of all methods and just methods calculated with more than one beat.

\section{Discussion}

Currently, the voltage is calculated based on taking the $\mathrm{p} 2 \mathrm{p}$ value of one beat of the signal for each point. This 
along with a threshold is then used to determine the LVA. Therefore, the question arose, if one beat is sufficient to calculate the voltage or if all the beats within the measured time segment should be used. From the results, it can be seen that taking one beat can be sufficient but it depends on which beat is taken, for example using the max or min $\mathrm{p} 2 \mathrm{p}$ values may have outliers and not give a true representation of the voltage at that point. Additionally, the same situation may occur when using the 75th percentile method, if the number of beats used is too small. For the CARTO method, only $30 \%$ of points had voltage values in a range of $0.1 \mathrm{mV}$ compared to the median. Therefore, showing that there may be many points in which an outlier beat may have been taken.

Additionally, the atrium was split into ten sections to further look at the inter-method variability with respect to the regions. For methods calculated with more than one beat, the variability was low, for most sections. However, the max and min method shows more variation between regions, which can be due to outliers being taken. The CARTO method shows the most variability, having an average voltage similar to that of the median method in some sections and the 75th percentile method in others. This indicates that it may not always be providing a true value for the voltage, rather choosing sometimes the outliers and others a peak which better represents the signal.

It can be seen that calculating the voltage based on taking a method which does not only take one beat could be a more appropriate method. However, a further investigation must be carried out on how the areas change on the map in regards to being classified as low/high voltage points with these new methods and if they can provide a higher correlation between voltage maps in SR and AF. Additionally, since only two patient were used for the analysis, it is unknown if these results are representative of all patients.

\section{Conclusion}

In this study, different methods for calculating the voltage were used and compared. It was seen that using a method which considers all beats within the measured time window rather than just one beat can give a better representation of the signal at each point. Additionally, there is little inter-variability between these methods for different regions of the atrium. Based on this study, it has been found that using the median $\mathrm{p} 2 \mathrm{p}$ method can provide an appropriate way to calculate the voltage which can help to correctly identify LVA in the map.

\section{Acknowledgments}

We gratefully acknowledge financial support by Deutsche Forschungsgemeinschaft (DFG) through D0637/ 22-3 and by the Ministerium für Wissenschaft, Forschung und Kunst Baden-Württemberg through the Research Seed Capital (RiSC) program

\section{References}

[1] Miyasaka Y, et al. Time trends of ischemic stroke incidence and mortality in patients diagnosed with first atrial fibrillation in 1980 to 2000: report of a community-based study. Stroke a Journal of Cerebral Circulation 2005;36(11):23622366.

[2] Oral H, et al. Pulmonary vein isolation for paroxysmal and persistent atrial fibrillation. Circulation 2002;105(9):10771081.

[3] Verma A, et al. Pre-existent left atrial scarring in patients undergoing pulmonary vein antrum isolation: an independent predictor of procedural failure. Journal of the American College of Cardiology 2005;45(2):285-292.

[4] Magnani S, et al. Adjunct ablation strategies for persistent atrial fibrillation-beyond pulmonary vein isolation. Journal of Thoracic Disease 2015;7(2):178-184.

[5] Rolf S, et al. Tailored atrial substrate modification based on low-voltage areas in catheter ablation of atrial fibrillation. Circulation Arrhythmia and electrophysiology 2014; 7(5):825-33.

[6] Blandino A, et al. Left atrial substrate modification targeting low-voltage areas for catheter ablation of atrial fibrillation: A systematic review and meta-analysis. Pacing and clinical electrophysiology PACE 2017;40(2):199-212.

[7] Ammar-Busch S, et al. Predictors of low voltage areas in persistent atrial fibrillation: is it really a matter of time? Journal of interventional cardiac electrophysiology an international journal of arrhythmias and pacing 2018;

[8] Rodríguez-Mañero M, et al. Validating left atrial low voltage areas during atrial fibrillation and atrial flutter using multielectrode automated electroanatomic mapping. JACC Clinical Electrophysiology 2018;4(12):1541-1552.

[9] Pan J, Tompkins WJ. A real-time QRS detection algorithm. IEEE Transactions on Biomedical Engineering 1985; 32(3):230-236.

[10] Nguyen M, et al. A new approach for automated location of active segments in intracardiac electrograms. IFMBE Proceedings World Congress on Medical Physics and Biomedical Engineering 2009;25/4:763-766.

[11] Jadidi A, et al. Inverse relationship between fractionated electrograms and atrial fibrosis in persistent atrial fibrillation: combined magnetic resonance imaging and highdensity mapping. Journal of the American College of Cardiology 1 2013;62(9):802-812.

Address for correspondence:

Deborah Nairn

Institute of Biomedical Engineering, Karlsruhe Institute of Technology (KIT), Fritz-Haber-Weg 1, 76131 Karlsruhe, Germany publications@ibt.kit.edu 